\title{
Microstructured induced band pattern in Love wave propagation for novel non destructive testing (NDT) procedures
}

\begin{abstract}
We propose a new approach for assessing microstructural properties of materials via nondistructive testing (NDT). This approach lies on the observation that, accounting for the microstructure within the materials, reveals a nonclassical band propagation pattern for Love waves. Precisely this propagation structure may be directly related to the internal microstructure. To illustrate this, propagation of Love waves is first investigated within the linear theory of couple stress materials with micro-inertia. Proving wave existence by the argument principle provides a closed-form condition for propagation to occur. This connection defines propagation bands, whose limits correspond to the situation when Love waves move with the same speed as bulk waves in the underlying half-space (internal resonance). This condition is closely related to the layer-to-substrate microstructure and it may be used to assess either of the two. Furthermore, we show that the frequency equation is a three-term combination of antiplane Rayleigh and Rayleigh-Lamb functions (in a free and in a free/clamped plate). Consequently, investigation of any extra observable, such as Rayleigh waves, reduces the risk of multiple solutions at the signal processing stage. We finally consider the limit as either the half-space or the layer becomes classical elastic. We show that this unseemly bonding of dissimilar models, sometimes adopted in the literature, usually leads to inconsistencies.
\end{abstract}

Keywords: Love waves, Non destructive testing, Pass-bands, Internal resonance 


\section{Introduction}

1 Love waves are antiplane waves localized near the free surface of a layer in 2 perfect contact with a half-space. These celebrated waves were first considered 3 by Love (1911) in a timely attempt to explain the defying appearance of an4 tiplane Rayleigh waves in seismograms, something that could not be achieved

5 in a homogeneous solid by the classical theory (Maugin, 1988; Gourgiotis and 6 Georgiadis, 2015). The absence of a wave component normal to the surface 7 makes Love waves especially attractive for NEMS and ultrasonic transducers

s (Jin et al. 2005). Although Love waves have been studied in great detail in 9 the context of classical elasticity (Achenbach, 1984, Graff, 1991), no investigation is available that performs this analysis in the context of complex materials, namely material models which account for microstructure. Besides, in recent years there has been an increased interest in Love waves as potential candidates for developing new non destructive testing (NDT) procedures, see Destuynder and Fabre (2016) and references therein. Indeed, this approach falls in the wake of existing successful applications in the field of defect detection for piezoelectric ceramics (Jin et al. 2005). Also, consideration of multiple observables, beyond the traditional Rayleigh waves, is capable of overcoming the well-known issue of solution non-uniqueness at the signal post-processing stage, cf.Dal Moro (2020).

Couple stress theory is perhaps the simplest strain-gradient theory and it aims to account for the discrete nature of materials at the micro-scale (Mindlin, 1964). Indeed, incorporating microstructural features inside classical elasticity is a complex and yet important feat, for it allows to remediate many drawbacks of the original theory. Among these, we mention the non-dispersive feature of bulk and Rayleigh waves, the absence of antiplane Rayleigh waves, the impossibility to predict a size for shear bands and the unbounded nature of stress near defects, as in crack problems. A number of papers have investigated the way microstructure alters such outcomes, through consideration of different complex materials in the form of strain-gradient, micropolar, surface and non-local elasticity. In their pioneering work, Graff and Pao (1967) studied plane strain 
reflection of waves impinging onto a free surface of a couple stress solid in the absence of micro-inertia. They proved that waves propagate dispersively and possibly faster than Rayleigh waves. Shortly later, Sengupta and Ghosh (1974) investigated wave propagation in a couple stress layer. The first recognition that support of antiplane localized waves may be granted by "perturbation" of the classical boundary conditions is due to Maugin (1988). In particular, Vardoulakis and Georgiadis (1997) showed that introducing microstructural characteristics in the form of strain-gradient and surface-energy terms allows the theory to support antiplane Rayleigh waves only when accounting for a certain type of gradient anisotropy. Ottosen et al. (2000) considered dispersion of Rayleigh waves in microstructured media which are described by the couple stress theory without micro-inertia. This work has been later extended by Georgiadis and Velgaki (2003), where a number of insightful remarks are given with regard to the importance of considering rotational inertia for successfully reproducing results from lattice theories. Gourgiotis and Georgiadis (2015) show that antiplane Rayleigh and torsional waves are supported in a homogeneous half-space when a complete strain gradient theory is considered. Recently, Nobili et al. (2020) proved existence of a novel antiplane evanescent wave arising by mode conversion in couple stress elasticity with micro-inertia. It is precisely this wave that has been seen radiating energy away in the dynamic loading of a crack (Nobili et al. 2019).

The analysis is further complicated when two or more bodies are set in contact. Li et al. (2018) study reflection and transmission of plane strain thermoflastic coupled waves in couple stress materials without micro-inertia. Wang et al. (2017) investigate plane strain reflection and transmission of elastic waves impinging onto a layer glued in between two couple stress half-spaces disregarding rotational inertia. Results are very involved and the limiting case of classical elastic half-spaces is also addressed. Recently, Nobili et al. (2021) considered propagation of Stoneley waves at the boundary between two couple stress halfspaces and found that incorporation of the microstructure greatly relaxes the classical restrictions for wave existence. In this paper, we show that this fea- 
ture may be put to advantage to relate the band propagation structure to the underlying material microstructure.

We also address the not trivial situation where a couple stress body is bonded to a classical solid. Several contributions are available in the literature dealing with such layout. As an example, Fan and $\mathrm{Xu}(2018)$ considered Love waves arising on the surface of a couple stress layer bonded to a classical half-space, while Ray and Singh (2020) studied a couple stress stratum imperfectly bonded to a viscoelastic substrate. Since couple stress entails a kinematical description that is richer than classical elasticity's, the question of what boundary conditions are to be imposed is not trivial. The matter is settled in this paper by taking the proper limit of the general solution for Love waves localized in a couple stress layer perfectly bonded to a couple stress half-space in the presence of micro-inertia.

\section{Variational derivation of couple stress elasticity}

We begin by considering microstructural features within our description of elastic materials. For this, as it occurs in polar materials, we supplement the classical displacement field $\boldsymbol{u}$ by the micro-rotation field $\boldsymbol{\varphi}$ as the kinematical fundamental variables. However, in contrast to Cosserat micro-polar theories, wherein displacements and micro-rotations are independent fields, couple stress (CS) theory relates one to the other, through the connection

$$
\varphi=\frac{1}{2} \operatorname{curl} \boldsymbol{u}
$$

Component-wise, this reads $\varphi_{i}=\frac{1}{2} E_{i j k} u_{k, j}$, where $i, j, k \in\{1,2,3\}, E_{i j k}$ is the alternator tensor and Einstein's summation convention on twice repeated subscripts is assumed. Hereinafter, a subscript comma denotes partial differentiation, e.g. $\left(\operatorname{grad} u_{k}\right)_{j}=u_{k, j}=\partial u_{k} / \partial x_{j}$, while subscript round brackets produce symmetrization, i.e. $u_{(i, j)}=\left(u_{i, j}+u_{j, i}\right) / 2$. Alongside the linear strain tensor $\varepsilon$ commonly defined in classical elasticity (CE)

$$
\varepsilon_{i j}=u_{(i, j)},
$$


we introduce the torsion-flexure (wryness or curvature) tensor $\chi$

$$
\chi_{i j}=\varphi_{i, j}
$$

s8 that, in light of the connection (1), is purely deviatoric, i.e. $\chi=\chi^{D}$, being sя $\chi^{D}=\chi-\frac{1}{3}(\operatorname{tr} \chi) \mathbf{1}$, where $\mathbf{1}$ is the rank-2 identity tensor and $\operatorname{tr} \boldsymbol{\chi}=\boldsymbol{\chi} \cdot \mathbf{1}$

$93[0, t]$

$$
\mathcal{A}=-\int_{0}^{t} \mathcal{L} \mathrm{d} \tau
$$

having introduced the Lagrangian function $\mathcal{L}=\mathcal{K}-\mathcal{V}$ as the difference between the kinetic and the potential energy. For the former, we take

$$
\mathcal{K}=\int_{\mathcal{B}}\left(\frac{1}{2} \rho \dot{\boldsymbol{u}}^{2}+\frac{1}{2} J \dot{\varphi}^{2}\right) \mathrm{d} V
$$

where $\rho$ and $J$ are the mass- and the micro-inertia densities, respectively. Hereinafter, a superposed dot denotes time differentiation, i.e. $\dot{\boldsymbol{u}}=\partial \boldsymbol{u} / \partial t$. In the absence of body forces, the potential energy reads

$$
\begin{aligned}
\mathcal{V}=\int_{\mathcal{B}}\left[\frac{1}{2} \boldsymbol{\sigma} \cdot \boldsymbol{\varepsilon}+\frac{1}{2} \boldsymbol{\mu}^{T} \cdot \boldsymbol{\chi}+\boldsymbol{\pi} \cdot\left(\boldsymbol{\varphi}-\frac{1}{2} \operatorname{curl} \boldsymbol{u}\right)\right] \mathrm{d} V & \\
& -\int_{\partial \mathcal{B}}\left(\boldsymbol{p}_{\boldsymbol{n}} \cdot \boldsymbol{u}+\boldsymbol{q}_{\boldsymbol{n}} \cdot \boldsymbol{\varphi}\right) \mathrm{d} A,
\end{aligned}
$$

94 and the superscript $T$, denoting transposition, is introduced for compatibility with Koiter (1964). The surface integral in (4) accounts for externally applied force and couple stress tractions, respectively $\boldsymbol{p}_{\boldsymbol{n}}$ and $\boldsymbol{q}_{\boldsymbol{n}}$.

As in $\mathrm{CE}$, conjugated to the strain tensor $\varepsilon$ is the Cauchy (or force) stress tensor, $\boldsymbol{t}$, which is generally non-symmetric. Consequently, it may be decomposed into its symmetric and skew-symmetric part, respectively

$$
\sigma_{i j}=t_{(i j)}, \quad \tau_{i j}=t_{i j}-\sigma_{i j}
$$

97 Since $\varepsilon$ is symmetric, only $\boldsymbol{\sigma}$ really performs work. 
Conjugated to the curvature tensor $\chi$ is the couple stress tensor $\boldsymbol{\mu}$, which, in general, performs work through all its components. However, it should be noted that $\boldsymbol{\chi}$ is deviatoric, whence only the deviatoric part of $\boldsymbol{\mu}^{D}$ really matters. As a result, to any effect, $\boldsymbol{\mu}$ may be replaced by its deviatoric part $\boldsymbol{\mu}^{D}$. Indeed, this theory is sometimes named indeterminate after the observation that the first invariant of the couple stress tensor rests indeterminate. Consequently, it may be set equal to zero without any loss of generality, e.g. $\operatorname{tr} \boldsymbol{\mu}=0$. For the sake of brevity, in the following we shall write $\boldsymbol{\mu}$, with the understanding that $\boldsymbol{\mu}^{D}$ is meant. For any surface of unit normal $\boldsymbol{n}$, the tensor $\boldsymbol{\mu}$ determines the internal reduced couple vector $\overline{\boldsymbol{q}}=\boldsymbol{\mu}^{T} \boldsymbol{n}$ acting across that surface.

Finally $\pi$ is a Lagrange multiplier enforcing the kinematical constraint (1) and allowing $\boldsymbol{u}$ and $\boldsymbol{\varphi}$ to be varied independently. For it, we let $\boldsymbol{\pi}=-2$ axial $\boldsymbol{\tau}$, with $(\text { axial } \tau)_{i}=\frac{1}{2} E_{i j k} \tau_{j k}$ denoting the axial vector attached to the skewsymmetric stress $\boldsymbol{t}$ (in Nobili et al. (2020) we dispensed with the factor 2 in the definition of the axial vector). With this definition, we have

$$
\text { axial } \boldsymbol{\tau}=\left[\tau_{23}, \tau_{31}, \tau_{12}\right]
$$

and the inverse formula $E_{i j k}(\operatorname{axial} \tau)_{k}=\tau_{i j}$.

Component-wise, the Lagrangian volumetric density reads

$$
\mathcal{L}=\frac{1}{2} \rho \dot{u}_{i} \dot{u}_{i}+\frac{1}{2} J \dot{\varphi}_{i} \dot{\varphi}_{i}-\frac{1}{2} \sigma_{i j} u_{i, j}-\frac{1}{2} \mu_{j i} \varphi_{i, j}-\pi_{i}\left(\varphi_{i}-\frac{1}{2} e_{i j k} u_{k, j}\right)
$$

By Hamilton's principle, the problem's governing equations are the Euler-Lagrange equations for the action integral. In the absence of body forces, they read

$$
\begin{aligned}
\operatorname{div} \boldsymbol{t} & =\rho \ddot{\boldsymbol{u}}, \\
2 \operatorname{axial} \boldsymbol{\tau}+\operatorname{div} \boldsymbol{\mu} & =J \ddot{\varphi},
\end{aligned}
$$

it being $\operatorname{div} \boldsymbol{t}=t_{j i, j}$. Application of the permutation tensor to Eq.5b yields

$$
\boldsymbol{\tau}=-\frac{1}{2} \mathbf{E}(\operatorname{div} \boldsymbol{\mu}-J \ddot{\varphi})
$$

whence the skew-symmetric part of the force stress tensor $\boldsymbol{t}$ is determined by rotational equilibrium, beside any constitutive consideration. It follows that 
Eq. (6) is generally not objective (Ottosen et al., 2000, Gourgiotis et al., 2013), although, for time-harmonic motions, this is no longer an issue (Shodja et al. 2015).

\subsection{Antiplane deformations}

Under antiplane deformations, the displacement field $\boldsymbol{u}=\left[u_{1}, u_{2}, u_{3}\right]$ is completely defined by the out-of-plane component $u_{3}=u_{3}\left(x_{1}, x_{2}, t\right)$. Then, the nonzero components of the micro-rotation vector, of the strain and of the curvature tensor become (see Nobili et al. (2019, 2020, 2021))

$$
\begin{array}{rlrl}
\varphi_{1} & =\frac{1}{2} u_{3,2}, & \varphi_{2} & =-\frac{1}{2} u_{3,1}, \\
\varepsilon_{13} & =\frac{1}{2} u_{3,1}, & & \\
\varepsilon_{23} & =\frac{1}{2} u_{3,2}, \\
\chi_{11} & =-\chi_{22}=\frac{1}{2} u_{3,12}, & \chi_{21} & =-\frac{1}{2} u_{3,11},
\end{array}
$$

Consequently, Eqs.(5) simplifies to

$$
\begin{aligned}
\sigma_{13,1}+\sigma_{23,2}+\tau_{13,1}+\tau_{23,2} & =\rho \ddot{u}_{3}, \\
\mu_{11,1}+\mu_{21,2}+2 \tau_{23} & =J \ddot{\varphi}_{1}, \\
\mu_{12,1}+\mu_{22,2}-2 \tau_{13} & =J \ddot{\varphi}_{2} .
\end{aligned}
$$

For hyperelastic materials, a stored energy potential $U=U(\varepsilon, \chi)$ exists which connects the strain $\varepsilon$ and the curvature $\chi$ to the Cauchy stress and to the couple stress. Indeed, we have

$$
\boldsymbol{\sigma}=\frac{\partial U}{\partial \varepsilon}, \quad \boldsymbol{\mu}^{T}=\frac{\partial U}{\partial \chi}
$$

which, to leading order for small deformations of an isotropic material, yield

$$
\boldsymbol{\sigma}=2 G \boldsymbol{\varepsilon}+\Lambda(\operatorname{tr} \boldsymbol{\varepsilon}) \mathbf{1}, \quad \boldsymbol{\mu}=2 G l^{2}\left(\chi^{T}+\eta \chi\right)
$$

117 Here, $\Lambda$ and $G>0$ are the classical Lamé moduli, with $3 \Lambda+2 G>0$. Also, $l>0$ is a characteristic length connected to the microstructure and $-1<\eta<1$

is a dimensionless number similar to Poisson's ratio. The material parameters $l$ and $\eta$ may be determined experimentally as in Lakes (1986). The situation $\eta=0$ corresponds to the strain gradient effect considered in Zhang et al. (1998), 
while the limiting value $\eta=1$ corresponds to the modified couple stress theory of elasticity introduced in Yang et al. (2002) via the balance of torques of torques.

The constitutive equations (9), in light of the definitions (2 3) and with the help of the kinematic relations (7), give the force stress and the couple stress in terms of the $u_{3}$ displacement alone (Nobili et al. 2019)

$$
\begin{array}{ll}
\sigma_{13}=G u_{3,1}, & \sigma_{23}=G u_{3,2}, \\
\mu_{11}=-\mu_{22}=G l^{2}(1+\eta) u_{3,12}, & \mu_{21}=G l^{2}\left(u_{3,22}-\eta u_{3,11}\right), \\
\mu_{12}=-G l^{2}\left(u_{3,11}-\eta u_{3,22}\right) . &
\end{array}
$$

Introducing Eqs. (7a 10$)$ into (6) yields

$$
\tau_{13}=-\frac{1}{2} G l^{2} \hat{\triangle} u_{3,1}+\frac{J}{4} \ddot{u}_{3,1}, \quad \tau_{23}=-\frac{1}{2} G l^{2} \hat{\triangle} u_{3,2}+\frac{J}{4} \ddot{u}_{3,2},
$$

which correspond to Eqs.(9) of Mishuris et al. (2012). Here, $\hat{\triangle}$ denotes the 2-D Laplace operator in the dimensional co-ordinates $\left(x_{1}, x_{2}\right)$. Finally, Eqs. 10a and (11) allow to rewrite translational equilibrium (8a) as the meta biharmonic equation

$$
G\left(\frac{1}{2} l^{2} \hat{\triangle} \hat{\triangle} u_{3}-\hat{\triangle} u_{3}\right)-\frac{J}{4} \hat{\triangle} \ddot{u}_{3}+\rho \ddot{u}_{3}=0 .
$$

\subsection{Boundary conditions}

Accounting for surface terms in Hamilton's principle gives the boundary conditions

$$
\boldsymbol{p}=\boldsymbol{p}_{\boldsymbol{n}}, \quad \text { and } \quad \boldsymbol{q}=\boldsymbol{q}_{\boldsymbol{n}},
$$

where we have let the reduced traction and reduce couple stress vectors, respectively

$$
\begin{aligned}
& \boldsymbol{p}=\boldsymbol{t}^{T} \boldsymbol{n}+\frac{1}{2} \operatorname{grad} \mu_{n n} \times \boldsymbol{n}, \\
& \boldsymbol{q}=\boldsymbol{\mu}^{T} \boldsymbol{n}-\mu_{n n} \boldsymbol{n} .
\end{aligned}
$$

Here, $\mu_{n n}=\boldsymbol{n} \cdot \boldsymbol{\mu} \boldsymbol{n}$ is the normal component of the couple stress traction vector.

Eq. 13b shows that only the tangential part of the couple stress vector may be prescribed. Indeed, it can be proved that the normal component of the couple stress tension is precisely annihilated by the second term in $13 \mathrm{a}$, see also Koiter (1964) and Ottosen et al. (2000). 


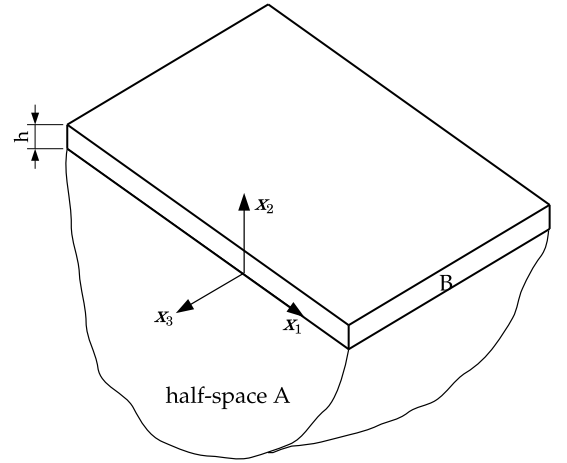

Figure 1: An infinite layer (B) perfectly bonded to an elastic half-space (A). The half-space is often named the substrate

\subsection{Nondimensionalization and time harmonic motions}

Let us consider a Cartesian co-ordinate system $\left(O, x_{1}, x_{2}, x_{3}\right)$ and a layer, $\mathcal{B}_{B}=\left\{\left(x_{1}, x_{2}, x_{3}\right): 0<x_{2}<h\right\}$, in perfect contact with the half-space $\mathcal{B}_{A}=\left\{\left(x_{1}, x_{2}, x_{3}\right): x_{2}<0\right\}$, see Fig 1 . The layer and the half-space are made of generally different isotropic elastic couple stress (CS) materials, for which antiplane deformations are considered.

At the layer top face, $x_{2}=h$, it is $\boldsymbol{n}=[0,1,0]$ and, according to Eqs.113, the out-of-plane component of the reduced force traction and the in-plane components of the couple stress traction read, respectively,

$$
p_{3}=\left(t_{23}+\frac{1}{2} \mu_{22,1}\right), \quad \text { and } \quad q_{1}=\mu_{21}, q_{2}=0 .
$$

We introduce the reference length $\Theta l$, by which we scale the spatial coordinates $\left(\xi_{1}, \xi_{2}, \xi_{3}\right)=\left(x_{1}, x_{2}, x_{3}\right)(\Theta l)^{-1}$ and the layer thickness $H=h / l$. In the same manner, we let the reference time $T=l / \tilde{c}_{s}$ and the dimensionless time $\tau=t / T$. Here, $\tilde{c}_{s}=\sqrt{G / \rho}$ is the bulk shear wave speed of CE. The parameter $\Theta$ is a convenient factor that is introduced so as to simplify notation. The equilibrium equation 12 becomes

$$
\triangle u_{3}-2 \Theta^{2} \triangle u_{3}-2 \Theta^{4}\left(\frac{\ell^{2}}{\Theta^{2}} \triangle u_{3, \tau \tau}-u_{3, \tau \tau}\right)=0,
$$

where $\triangle$ is the 2-D Laplace operator in $\xi_{1}$ and $\xi_{2}$ and we have let the dynamic 
151

characteristic length (Mishuris et al. 2012)

$$
\ell=\frac{l_{d}}{l}, \quad \text { with } \quad l_{d}=\frac{1}{2} \sqrt{\frac{J}{\rho}}
$$

152

The latter is proportional to $2 \sqrt{6} l_{d}$, that is introduced in Shodja et al. (2015).

Consideration of time-harmonic solutions brings

$$
u_{3}=W\left(\xi_{1}, \xi_{2}\right) \exp (-\imath \Omega \tau)
$$

where $\imath$ is the imaginary unit and $\Omega=\omega T>0$ the dimensionless (time) frequency. Then, Eq. 15 yields the meta biharmonic PDE Georgiadis and Velgaki, 2003, Eq.(19)) for the wave amplitude $W$ :

$$
\left[\triangle \triangle-2\left(1-\ell^{2} \Omega^{2}\right) \Theta^{2} \triangle-2 \Omega^{2} \Theta^{4}\right] W=0 .
$$

This homogeneous equation is easily factored

$$
\left(\triangle+\delta^{2}\right)(\triangle-1) W=0
$$

provided that $\Theta$ is let as in (Nobili et al. 2020, Eq.(3.4)

$$
\Theta^{2}=\frac{\sqrt{\left(1-\ell^{2} \Omega^{2}\right)^{2}+2 \Omega^{2}}-1+\ell^{2} \Omega^{2}}{2 \Omega^{2}}
$$

159 We then have (Nobili et al., 2021, Eq.(21))

$$
\delta=2 \delta_{c r} \Theta^{2}, \quad \text { with } \quad \delta_{c r}=\ell_{c r} \Omega, \quad \ell_{c r}=1 / \sqrt{2} .
$$

Eq. 17 is especially convenient for it shows that two (antiplane) bulk modes are supported: one, travelling, having wavenumber $\kappa= \pm \delta$, and another, evanescent, possessing wavenumber $\kappa= \pm \imath$ (Nobili et al., 2020).

In dimensionless form, the traction vectors 14 become

$$
\begin{aligned}
p_{3} & =-\frac{G}{2 \Theta^{3}}\left[\left(\delta^{2}-1\right) W_{, 2}+(\eta+2) W_{, 112}+W_{, 222}\right], \\
q_{1} & =\frac{G l}{\Theta^{2}}\left(W_{, 22}-\eta W_{, 11}\right) .
\end{aligned}
$$




$$
\ell_{A, B}=l_{d A, B} / l_{A, B}
$$

176 being $l_{d A, B}=\frac{1}{2} \sqrt{J_{A, B} / \rho_{A, B}}$. Besides, we define the equivalent of $\delta$ for $\mathrm{B}$, 177 cf.(Nobili et al., 2021, Eq.(24))

$$
\frac{\psi}{v}=\frac{\sqrt{\left(1-\ell_{B}^{2} \Omega_{B}^{2}\right)^{2}+2 \Omega_{B}^{2}}-1+\ell_{B}^{2} \Omega_{B}^{2}}{\sqrt{2} \Omega_{B}} .
$$


Indeed, $\psi=\delta$ when $\ell_{B}=\ell_{A}$ and $v=1$. Also, $\delta \sim \psi \sim \Omega / \sqrt{2}$ and $\Theta \sim 2^{-1 / 2}$ as $\Omega \rightarrow 0$. For $\psi$ we have the asymptotics

$$
\psi \rightarrow \sqrt{2} \Omega \ell_{B}+O\left(v^{2}\right), \quad \text { as } \quad v \rightarrow 0,
$$

and

$$
\psi \rightarrow \delta_{c r}+O\left(v^{-2}\right), \quad \text { as } \quad v \rightarrow+\infty .
$$

The governing equation (16) specializes to

$$
\left(\triangle+\delta^{2}\right)(\triangle-1) W_{A}=0, \quad\left(\xi_{1}, \xi_{2}\right) \in \mathcal{B}_{A},
$$

for the half-space A, and to

$$
\left(\triangle+\delta_{1}^{2}\right)\left(\triangle-\delta_{2}^{2}\right) W_{B}=0, \quad\left(\xi_{1}, \xi_{2}\right) \in \mathcal{B}_{B},
$$

for the layer B. Clearly, $W_{A}$ and $W_{B}$ are the solution amplitudes in medium A and $\mathrm{B}$, respectively. Also, we have let the dimensionless wavenumbers

$$
\delta_{1,2}=\frac{\kappa_{1,2}}{\beta}, \quad \text { with } \quad \kappa_{1}=\frac{\sqrt{\delta \psi}}{v}, \kappa_{2}=\sqrt{\delta / \psi},
$$

normalized with respect to $\Theta \ell_{A}$ and $\Theta \ell_{B}$, respectively. They correspond to bulk travelling and evanescent modes for B. With this notation, we can easily express the corresponding bulk wave phase speed with respect to $\tilde{c_{s}}$ or $\tilde{c_{s}}$

$$
c_{S H}=V_{A, B} \Theta \tilde{c_{s}, B},
$$

where we have let the dimensionless phase speed

$$
V_{A}=\frac{\Omega}{\delta}, \quad \text { and } \quad V_{B}=\frac{\Omega_{B}}{\kappa}=\frac{\Omega}{v \kappa} .
$$

34 In fact, we see that, in the limit 21], $V_{B}=\Theta^{-1}$ for $\kappa=\kappa_{1}$, which gives the classical bulk wavespeed $c_{S H}=\tilde{c_{s B}}$. For $\kappa=\kappa_{2}, V_{B} \rightarrow 0$ and the evanescent bulk mode becomes standing, as already pointed out in Nobili et al. 2019). We have the Short-Wave High-Frequency (SWHF) approximation for bulk waves

$$
\kappa^{2}-2 \ell_{A}^{4} \Omega^{2}=0
$$


For material B, the boundary tractions 20 become (Nobili et al. 2021 Eqs. (27))

$$
\begin{aligned}
& p_{3 B}=-\frac{G_{B}}{2 \Theta^{3}}\left\{\beta^{2}\left[\left(\eta_{B}+2\right) W_{B, 112}+W_{B, 222}\right]+\frac{\delta}{\psi}\left(\frac{\psi^{2}}{v^{2}}-1\right) W_{B, 2}\right\}, \\
& q_{1 B}=\frac{G_{B} l_{B}}{\Theta^{2}} \beta^{2}\left(W_{B, 22}-\eta_{B} W_{B, 11}\right),
\end{aligned}
$$

which, in the limiting case (21) and using of Eqs. 19 23), lends the classical limit.

\section{Dispersion relation for Love waves}

For guided propagation along $\xi_{1}$, we have (recall we chose $l=l_{A}$ )

$$
W_{A, B}\left(\xi_{1}, \xi_{2}\right)=l w_{A, B}\left(\xi_{2}\right) \exp \left(\imath \kappa \xi_{1}\right)
$$

where $K=k l$ denotes the dimensionless (spatial) wavenumber in the propagation direction $\xi_{1}$. Letting $V=\Omega / K$, we get the dimensional phase speed in the propagation direction

$$
c=\omega / k=V \tilde{c_{s A}} .
$$

The general decaying solution of Eq. 24), valid for the half-space A, reads

$$
w_{A}\left(\xi_{2}\right)=s_{1} \exp \left(A_{1} \xi_{2}\right)+s_{2} \exp \left(A_{2} \xi_{2}\right),
$$

where $s_{1,2}$ are undetermined amplitudes and

$$
A_{1}=\sqrt{\kappa^{2}-\delta^{2}}, \quad A_{2}=\sqrt{\kappa^{2}+1},
$$

are the decay indices in the thickness direction $\xi_{2}$. For decay to occur, we need to give proper and definite meaning to the square root multivalued function (Noble, 1958). This is obtained introducing the cut complex plane and choosing the particular branch of the square root such that $A_{1,2}(\kappa) \rightarrow|\kappa|$ as $\kappa \rightarrow \infty$ on the real axis (Nobili et al. 2019). Cuts start at the branch points $\pm \delta$ and move in opposing direction away from the real axis. As a result, $A_{1}(\kappa)$ is real positive on the real domain $|\kappa|>\delta$, and $A_{2}(\kappa)$ is real positive on the whole real axis. 
Similarly, we let

$$
B_{1}=\sqrt{\kappa^{2}-\delta_{1}^{2}}, \quad B_{2}=\sqrt{\kappa^{2}+\delta_{2}^{2}},
$$

and the general solution of Eq. 25) is given by Nobili et al. (2020)

$$
\begin{aligned}
w_{B}\left(\xi_{2}\right)=e_{1} \cosh \left(B_{1} \xi_{2}\right)+ & e_{2} \cosh \left(B_{2} \xi_{2}\right) \\
& +o_{1} B_{1}^{-1} \sinh \left(B_{1} \xi_{2}\right)+o_{2} B_{2}^{-1} \sinh \left(B_{2} \xi_{2}\right),
\end{aligned}
$$

$$
R_{A}(\kappa)=R_{0}\left(\kappa, A_{1}, A_{2}, \eta_{A}\right)
$$

to give the form already adopted in Nobili et al. (2019, 2020)

$$
\left(A_{1}-A_{2}\right) R_{A}(\kappa)=\zeta_{11 A}^{2} A_{1}-\zeta_{12 A}^{2} A_{2},
$$

having let $\zeta_{11 A}=\eta_{A} \kappa^{2}+A_{2}^{2}$ and $\zeta_{12 A}=\eta_{A} \kappa^{2}+A_{1}^{2}$.

\footnotetext{
${ }^{1}$ Provided that we replace $\ell$ with $\sqrt{2} \ell$ and take the opposite of $q^{2}$
} 


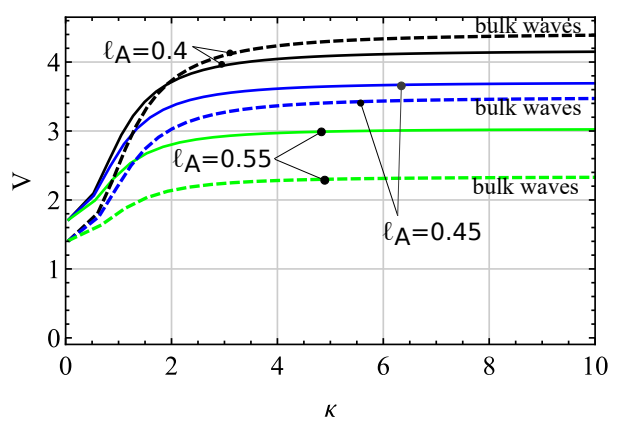

Figure 2: Dispersion curves for Rayleigh waves in medium B (solid) compared to bulk waves in medium $\mathrm{A}$ (dashed curves) with the parameters $\ell_{A}=0.4$ (black), $\ell_{A}=0.45$ (blue) and $\ell_{A}=0.55$ (green). It appears that only in the case $\ell_{A}=0.4$, bulk waves move faster than Rayleigh waves in the short-wave regime

Existence and uniqueness of antiplane Rayleigh waves is proved by the argument principle in Nobili et al. (2021), where it is also shown that the Rayleigh root is the single real zero of a bi-quartic polynomial equation which is regularly perturbed in $\eta_{A}$. Indeed, for $\eta_{A}=0$, we have $\kappa= \pm \delta_{1}$ whence, in general, we can write the solution as a power series in $\eta_{A}$

$$
\kappa_{R}^{2}=\delta_{1}^{2}\left(1+\frac{\delta_{1}^{6}}{\left(\delta_{1}^{2}+\delta_{2}^{2}\right)^{3}} \eta_{A}^{4}+\ldots\right) .
$$

This expansion, once specialized for A and up to first order correction terms in $\eta_{A}$, corresponds to (Nobili et al. 2020, Eq.(3.37)). From (32) follows the SWHF approximation

$$
\kappa^{2}-2 \Omega^{2} \frac{\ell_{A}^{2} \ell_{B}^{2}}{\beta^{2} v^{2}}\left(1+\eta_{A}^{4}+\ldots\right)=0,
$$

which provides the asymptotic limit for antiplane Rayleigh-Lamb (RL) modes.

Hereinafter, numerical exploration is presented for the parameter set $\ell_{B}=$ $0.5, \beta=v=1.1, H=0.1, \eta_{A}=0.8$ and $\eta_{B}=0.5$. Fig 2 plots the dispersion curves for Rayleigh waves in the layer B, superposed onto bulk waves in medium A. It appears that, when micro-inertia in A is small enough, bulk waves in A move faster than Rayleigh waves in B. 


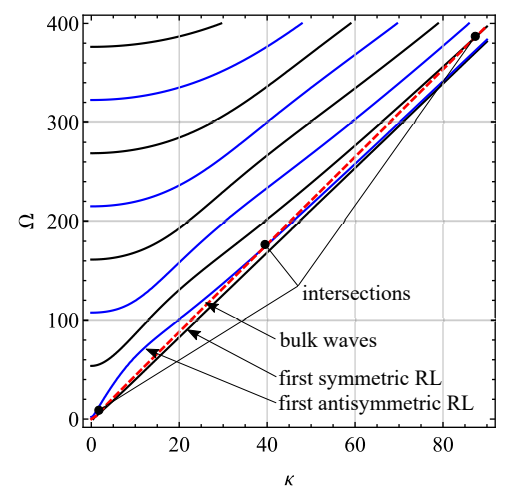

Figure 3: Frequency spectrum branches for symmetric (solid, black) and antisymmetric (solid, blue) Rayleigh-Lamb waves, with $\ell_{A}=0.4$. The spectrum of bulk waves in medium A (dashed, red) intersects RL modes in an infinite succession of points. These are cut-on/cut-off points for Lamb waves.

\subsection{Antiplane Rayleigh-Lamb waves in a free plate}

Guided propagation in the free layer is described by the RL frequency equation

$$
D_{R L}(\kappa)=d_{s}(\kappa) d_{o}(\kappa),
$$

where $d_{s}(\kappa)$ and $d_{o}(\kappa)$ are given in Nobili et al. (2020) respectively for symmetric and anti-symmetric modes. Looking at them, it is clear that $D_{R L}(\kappa)$ depends on $B_{1}$ and $B_{2}$ through even powers and therefore it is analytic in the whole complex plane, i.e. it is holomorphic. Physically, this means that there are no bulk waves associated to RL propagation. Eq 34 may be rewritten in terms of the Rayleigh function

$$
\begin{aligned}
D_{R L}(\kappa)=\frac{\left(B_{1}-B_{2}\right)^{2}}{8 B_{1} B_{2}} R_{B}(\kappa)^{2}\left[\cosh \left(\frac{H\left(B_{1}+B_{2}\right)}{\Theta}\right)-1\right] \\
-\frac{\left(\zeta_{11 B}^{2} B_{1}+\zeta_{12 B}^{2} B_{2}\right)^{2}}{8 B_{1} B_{2}}\left[\cosh \left(\frac{H\left(B_{1}-B_{2}\right)}{\Theta}\right)-1\right],
\end{aligned}
$$

whence we retrieve the well-known result that, when it comes to very short waves, the layer behaves just like a half-space.

Fig 3 plots the frequency spectrum for antiplane RL symmetric and antisymmetric waves in medium B, cfr.Nobili et al. (2020). In this Figure, RL branches are superposed onto the spectrum for $\mathrm{SH}$ bulk waves in medium A, and it ap- 

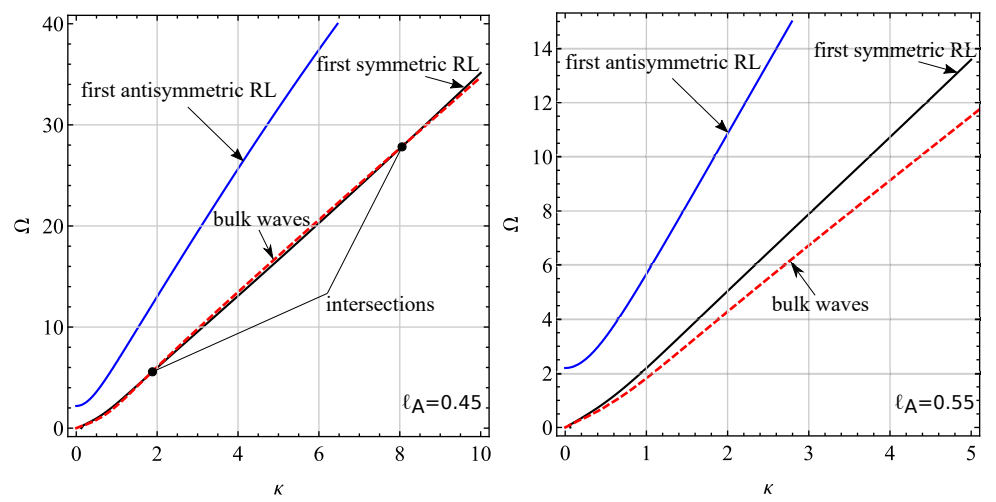

Figure 4: Frequency spectrum branches for symmetric (solid, black) and antisymmetric (solid, blue) Rayleigh-Lamb waves, with $\ell_{A}=0.45$ (left) and $\ell_{A}=0.55$ (right), superposed onto the spectrum of bulk waves in medium A (dashed, red). In the first case we have two intersections, in the second none.

pears that a infinite succession of intersection points occur, alternatively with symmetric and antisymmetric branches. These points are given by

$$
D_{R L}(\delta)=0,
$$

and represent propagation states for which RL waves in medium B coexist with bulk waves in medium A (or, equally, they have the same phase speed).

In contrast, when micro-inertia in medium A increases, we move to two intersections and, eventually, to none, as it is illustrated in Figs 4 . The two intersection condition is remarkable given that it occurs with the same first symmetric branch, i.e. there are two propagation frequencies that support longitudinal waves in the layer and also in the half-space. The regime shift from an infinite to a finite number of intersections occurs when the phase speed of Rayleigh waves in medium B becomes greater than that of bulk waves in medium A, as demonstrated in Fig.2. Therefore, comparing expansion (26) with (33), an approximate criterion for existence of an infinite number of intersections may be obtained

$$
\ell_{0 A}<\frac{\ell_{0 B}}{\beta v} .
$$

For example, with the parameter set of Fig 2 , we get $\ell_{0 A}<0.41$. This criterion may be improved for large values of $\eta_{A}$ by taking into consideration more terms 

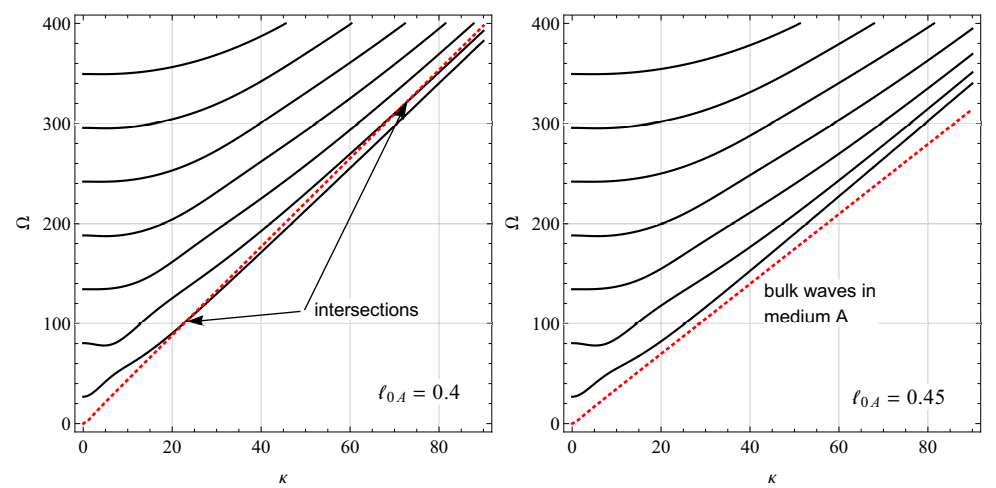

Figure 5: Frequency spectrum for Rayleigh-Lamb waves in a free/clamped plate, with $\ell_{A}=0.4$ (left) and $\ell_{A}=0.45$ (right). The frequency spectrum of bulk waves in medium $\mathrm{A}$ is also plotted (dotted, red) and it intersects RL modes only in the case $\ell_{A}=0.4$

in the expansions.

\subsection{Rayleigh-Lamb waves in a clamped-free plate}

Proceeding as in Nobili et al. (2020), it can be proved that propagation in a free/clamped (FC) layer occurs according to the holomorphic function

$$
\begin{gathered}
D_{F C}(\kappa)=\frac{1}{2} \frac{\left(B_{1}-B_{2}\right)^{2}}{B_{1} B_{2}} R_{B}(\kappa)\left[\cosh \left(\frac{H\left(B_{1}+B_{2}\right)}{\Theta}\right)-1\right]-\left(\zeta_{11 B}-\zeta_{12 B}\right)^{2} \\
-\frac{1}{2}\left(B_{2}^{-1}+B_{1}^{-1}\right)\left(\zeta_{11 B}^{2} B_{1}+\zeta_{12 B}^{2} B_{2}\right)\left[\cosh \left(\frac{H\left(B_{1}-B_{2}\right)}{\Theta}\right)-1\right] .
\end{gathered}
$$

In the SWHF approximation, the first term of $D_{F C}(\kappa)$ grows exponentially and dominates, whence propagation occurs through Rayleigh waves, just as in a half-space.

Fig 5 compares RL spectra in a free/clamped plate with bulk modes for medium A. Again, we see that an infinite succession of intersection points between the two is possible inasmuch as the phase speed of Rayleigh waves in medium $\mathrm{B}$ is lower than that of bulk waves in medium $\mathrm{A}$, which requirement is approximated by Eq. 36). Indeed, increasing rotational inertia in medium A, intersections are no longer possible, while a finite number of intersections (as it occurs for RL waves) is not supported. 
At the layer/half-space joining surface, we impose perfect adhesion

$$
\begin{aligned}
& w_{A}(0)=w_{B}(0), \\
& w_{A}^{\prime}(0)=w_{B}^{\prime}(0), \\
& p_{3 A}(0)=p_{3 B}(0), \\
& q_{1 A}(0)=q_{1 B}(0),
\end{aligned}
$$

while the layer top surface $\xi_{2}=H / \Theta$ is subjected to free conditions

$$
\begin{aligned}
& p_{3 B}\left(\Theta^{-1} H\right)=0, \\
& q_{1 B}\left(\Theta^{-1} H\right)=0 .
\end{aligned}
$$

Introducing the solutions 2830 into the boundary conditions $239 \mid 38$ yields a homogeneous system of linear equations in the unknown amplitudes $e_{1,2}, o_{1,2}, s_{1,2}$, which admits non-trivial solutions inasmuch as the secular (or frequency) equation

$$
\Delta(\kappa)=0
$$

255 is satisfied. Letting $\Gamma=G_{B} / G_{A}$, the ratio of the layer to the half-space shear moduli, the general form of the secular equation can be written as

$$
\Delta(\kappa)=\beta^{6} \Gamma^{2}\left(A_{1}-A_{2}\right) D_{0}(\kappa),
$$

257 having let the quadratic polynomial in $\Gamma$

$$
D_{0}(\kappa)=d_{0}+d_{1} \Gamma+d_{2} \Gamma^{2},
$$

with

$$
\begin{aligned}
& d_{0}=-\beta^{-2} R_{A}(\kappa) D_{F C}(\kappa), \\
& d_{1}=D_{1}(\kappa), \\
& d_{2}=-4 \beta^{2} D_{R L}(\kappa) .
\end{aligned}
$$


Here, $D_{1}(\kappa)$ expresses the coupling between the half-space and the layer

$$
\begin{aligned}
D_{1}(\kappa)= & \left(B_{1}-B_{2}\right) R_{B}(\kappa)\left\{c_{+}\left[\cosh \left(\frac{H\left(B_{1}+B_{2}\right)}{\Theta}\right)-1\right]\right. \\
& \left.-\frac{s_{+}}{B_{1}+B_{2}} \sinh \left(\frac{H\left(B_{1}+B_{2}\right)}{\Theta}\right)\right\}-\left(\zeta_{11 B}^{2} B_{1}+\zeta_{12 B}^{2} B_{2}\right) \times \\
\left\{c_{-}\right. & {\left.\left[\cosh \left(\frac{H\left(B_{1}-B_{2}\right)}{\Theta}\right)-1\right]-\frac{s_{-}}{B_{1}-B_{2}} \sinh \left(\frac{H\left(B_{1}-B_{2}\right)}{\Theta}\right)\right\}, }
\end{aligned}
$$

where

$$
\begin{aligned}
& c_{ \pm}=\left(\eta_{A} \kappa^{2}-A_{1} A_{2}\right) \frac{\zeta_{11 B} B_{1} \mp \zeta_{12 B} B_{2}}{B_{1} B_{2}}, \\
& s_{ \pm}= \pm \frac{1}{2}\left(B_{2}^{2}-B_{1}^{2}\right)\left(A_{1}+A_{2}\right)\left(B_{1} \pm B_{2}\right)\left(1 \pm \frac{A_{1} A_{2}}{B_{1} B_{2}}\right) .
\end{aligned}
$$

Rewriting Eq. 41 as in Appendix Appendix A.1 it is seen that dependence on $B_{1,2}$ occurs only through even powers, whence only cuts associated with $A_{1,2}$ remain.

When $A=B$, it is

$$
\left(\Gamma, \beta, v, \psi, \eta_{B}\right)=(1,1,1, \delta, \eta)
$$

and we retrieve the well expected result

$$
D_{0}^{A=B}(\kappa)=\left(\zeta_{11 A}-\zeta_{12 A}\right)^{2}\left(A_{1}-A_{2}\right) R_{A}(\kappa),
$$

whereby only Rayleigh waves propagate. Similarly, for an exceedingly weak layer, that is for $\Gamma \rightarrow 0$, we find either Rayleigh waves confined to the halfspace or RL waves trapped in the free/clamped layer, which bounce off the impenetrable rigid barrier posed by the half-space. In the special case of a vanishing layer, $H=0$, one gets, to leading order,

$$
D_{0}^{H=0}(\kappa)=\left(\zeta_{11 B}-\zeta_{12 B}\right)^{2}\left(A_{1}-A_{2}\right) R_{A}(\kappa),
$$

that gives Rayleigh waves again. Its classical limit 21] gives

$$
D_{0}^{H=0}(\kappa) \rightarrow-\frac{2 \delta^{2}}{\Omega^{2}}\left(\frac{c_{s B}}{c_{s A}}\right)^{4} B_{1},
$$

whence Rayleigh waves collapse onto travelling bulk SH waves. On the opposite side of the spectrum, for an exceedingly strong layer, that is for $\Gamma \rightarrow+\infty$, we obtain, as expected, RL modes. 


\section{Wave pattern and microstructural features}

We now consider the root landscape of $D_{0}(s)$ considered as a complex-valued function of the argument $s=\Re(s)+\imath \Im(s)$. Eq. (40) is noteworthy for it shows that the only branch cuts appearing in $D_{0}(s)$ are those brought by the Rayleigh function for the half-space A. Accordingly, in this system, only three families of waves may exist, namely:

1. Lamb waves, which are either travelling, when they correspond to the real zeros of $D_{0}$, or evanescent (Lamb-like waves), characterised by purely imaginary zeros of $D_{0}$;

2. bulk travelling waves, which move like SH bulk waves for the half-space A, possibly inhomogeneous: these are related to the branch cuts for $A_{1}(s)$;

3. bulk standing modes, related to the branch cuts for $A_{2}(s)$.

No bulk modes are possible that are related to SH bulk waves in B.

Existence and uniqueness of Love waves may be establish through the argument principle, following the procedure adopted in Cagniard (1962) and Nobili et al. (2021) for Stoneley waves, respectively in CE and CS. This technical and lengthy proof is sketched in Appendix A.2. One major result of this process is the necessary condition for the existence of Love waves, namely

$$
D_{0}(\delta) \geq 0
$$

equality setting the limits of the passbands, i.e. the cut-on and cut-off frequencies. Physically, the passband ends correspond to the situation when Love waves move with the same phase speed as bulk waves in medium A. In such states, energy is no longer trapped in the layer, bouncing back and forth between its boundaries, but leaks in the half-space in the form of bulk waves.

Eq. (42) is the fundamental result which enables to relates the band pattern to the microstructural features in the material. Also, several qualitative outcomes are possible in dependence of the ratio between the layer and the substrate microstructure, as it is presently described. 


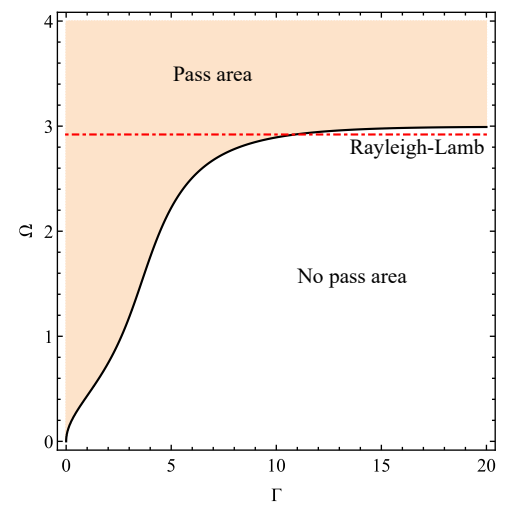

Figure 6: Cut-on frequency vs. shear stiffness ratio $\Gamma$ for $\ell_{A}=0.4<\ell^{B}=0.5$ (solid, black). Propagation occurs above the black curve, which asymptotes to the first symmetric RayleighLamb mode at the wavenumber $\kappa=\delta$ (red dash-dotted line).

\subsection{Infinite passbands}

Fig 6 plots the cut-on frequency as a function of $\Gamma$ for $\ell_{A}=0.4$, which, for large $\Gamma$, asymptotes to the first symmetric RL mode for a free plate at the wavenumber $\kappa=\delta$. Sweeping larger frequencies reveals a finite passband, that ranges from cut-on to cut-off, as it is shown in Fig7. The corresponding frequency spectrum for Love waves is shown in Fig 8 for $\Gamma=1$ and $\Gamma=5$ : the picture confirms that propagation only occurs within a finite frequency range. It also shows that the dispersive nature of propagation is restricted to low wavenumbers, a result which was already observed with respect to Rayleigh waves (Ottosen et al. 2000; Nobili et al., 2019).

From the discussion in Sec 3.2 we deduce that an infinite number of passbands is expected: for $\Gamma$ large, these are framed in between symmetric and antisymmetric RL modes. Similarly, for $\Gamma=0$, passbands are constrained by RL waves propagating in a clamped-free plate at $\kappa=\delta$, that is by the intersections indicated in Fig 5. In fact, the bounding curves for cut-on and cut-off start at $\Gamma=0$, which is realized provided that $D_{F C}(\delta)=0$.

\subsection{Single passband}

This propagation landscape, characterized by an infinite succession of passbands, holds only inasmuch as condition (36) stands. Indeed, when micro-inertia 


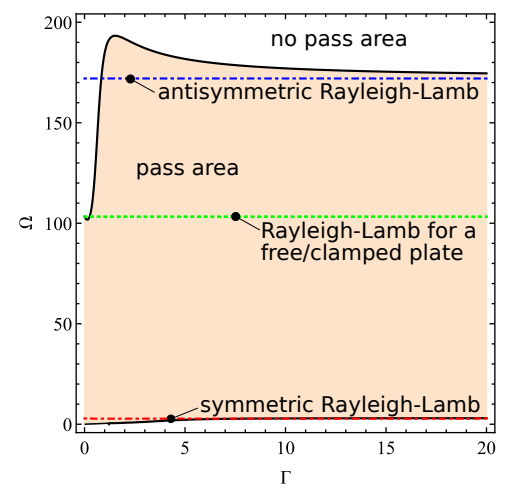

Figure 7: Passband frequency range vs. shear stiffness ratio $\Gamma$ (solid, black), with $\ell_{A}=0.4$. Propagation occurs in the (pink) region in between the black curves; these asymptote to the first symmetric/antisymmetric Rayleigh-Lamb frequencies, respectively for cut-on (red) and cut-off (blue dash-dotted lines). The cut-off curve sets off in correspondence to the occurrence of Rayleigh-Lamb waves in a free-clamped plate (green, dotted)
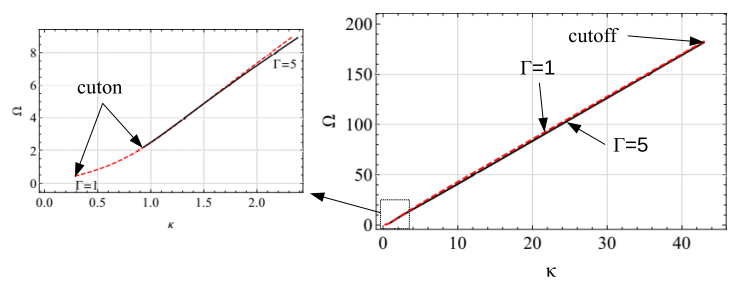

Figure 8: Frequency spectrum for $\Gamma=1$ (dashed, red) and $\Gamma=5$ (solid, black) with $\ell_{A}=$ $0.4<\ell^{B}=0.5$ : Lamb frequency spectra asymptote to Rayleigh waves

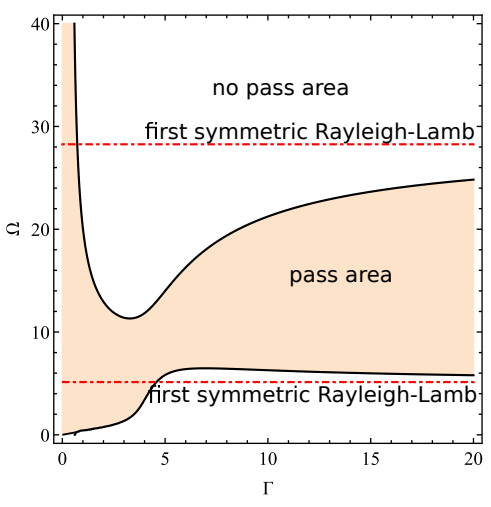

Figure 9: Cut-on frequency vs. shear stiffness ratio $\Gamma$ for $\ell_{A}=0.45$ (solid, black). Propagation occurs between the black curves, which asymptote to two frequencies in the first symmetric Rayleigh-Lamb branch at the wavenumber $\kappa=\delta$ (red dash-dotted lines). Since no intersection with RL waves in a free/clamped plate is possible, cut-off possesses a vertical asymptote 

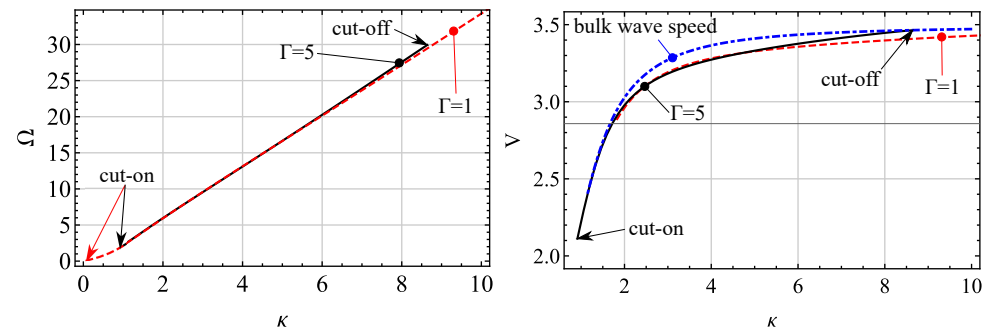

Figure 10: Frequency spectrum (left) for $\Gamma=1$ (dashed, red) and $\Gamma=5$ (solid, black), for $\ell_{A}=0.45$ : only for the latter cut-off is defined. Since spectra almost overlap, dispersion curves are also shown (right) and it is seen that cut-on and cut-off indeed occur at the intersections with the bulk wave speed (dash-dotted, blue)

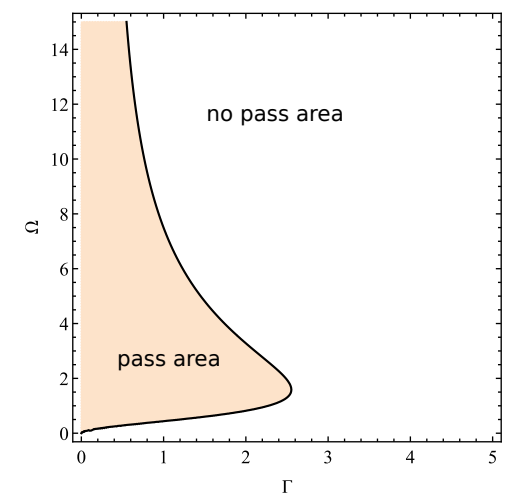

Figure 11: Cut-on frequency vs. shear stiffness ratio $\Gamma$ for $\ell_{A}=0.55>\ell_{B}=0.5$ (solid, black). Propagation occurs up to a limiting threshold for $\Gamma$.

of medium A is large enough (and therefore bulk waves move slow enough),

propagation occurs through a single passband, which asymptotes to a pair of frequencies along the first symmetric RL mode, as in Fig 4 . This situation is depicted in Fig 9, that plots cut-on and cut-off frequencies as a function of $\Gamma$ for $\ell^{A}=0.45$. Since no intersections of bulk waves with RL modes in a clamped/free plate are possible, cut-off displays a vertical asymptote and, as a consequence, for $\Gamma$ small enough, the passband is semi-infinite. The frequency spectrum in Fig 10 shows that this is indeed the case for $\Gamma=1$ and yet no longer for $\Gamma=5$.

\subsection{Love wave block-band}

Increasing further micro-inertia in material $\mathrm{A}$, an important change in the propagation features is encountered: namely the appearance of a bounding curve 


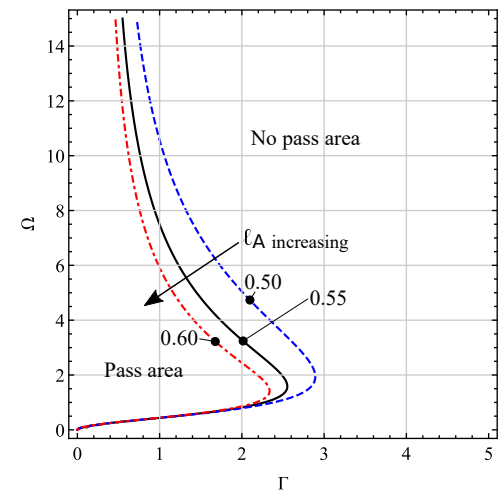

Figure 12: Cut-on frequency vs. shear stiffness ratio $\Gamma$ for $\ell_{A}=0.50$ (dashed, blue), 0.55 (solid, black) and 0.6 (dot-dashed, red): the pass region shrinks as rotational inertia increases in medium $\mathrm{A}$

for $\Gamma$. Indeed, since no RL modes in the layer are possible which equally support bulk waves in the half-space, the passband region becomes bounded by $\Gamma<$ $\Gamma_{M}(\Omega)$, as in Fig 11 . This passband region shrinks for larger values of rotational inertia, as illustrated in Fig 12

\section{The classical limits}

We now show that neglecting microstructure in only one of either the layer or the half-space generally leads to inconsistencies. Occasionally, however, a special set of boundary conditions is available which lends a well-posed problem.

\subsection{Classical layer perfectly bonded to a couple stress half-space}

We first consider the case when the layer B is classical elastic and the halfspace A is made of couple stress material. This condition is obtained by taking the limit 21) of the general case. We note that, as $\beta \rightarrow 0$,

$$
B_{1}=\sqrt{\kappa^{2}-\frac{\tilde{c_{s}}}{\tilde{c_{s B}}} \Omega^{2} \Theta^{2}}, \quad B_{2}=\frac{\sqrt{2} \Theta}{\beta}+O(1) .
$$

Then, we can solve Eq. $39 \mathrm{~b}$ for $o_{2}$ to obtain

$$
o_{2}=\left[-\frac{\sqrt{2} \Theta}{\beta} \operatorname{coth} \frac{\sqrt{2} H}{\beta}+O(\beta)\right] e_{2}+O\left(\frac{\beta}{\sinh (\sqrt{2} H / \beta)}\right) \text {, }
$$




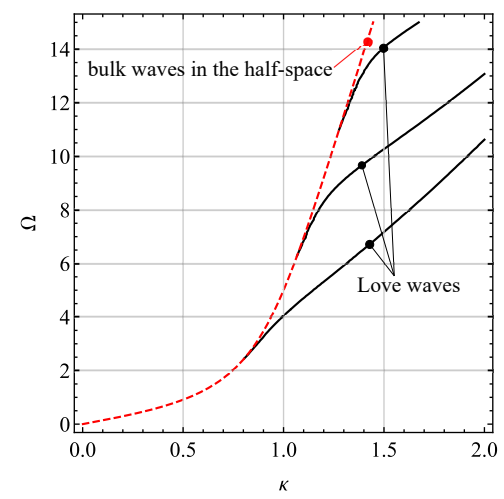

Figure 13: Love waves for a couple stress half-space in perfect contact with a classical layer (solid, black) for $H=1, \eta_{A}=0.5, \ell_{A}=0.2, \beta v=1.5$ and $\Gamma=3$. For each branch, cut-on is located in correspondence of bulk waves in the half-space (dashed, red).

where the last term is exponentially small. Consequently, rotation continuity (38b) reads

$$
o_{1}+o_{2}-A_{1} s_{1}-A_{2} s_{2}=0
$$

which, assuming all quantities to be $O(1)$, demands $e_{2}=O(\beta)$ to eliminate the $O\left(\beta^{-1}\right)$ term given by $o_{2}$. Solving the remaining equations in the system $[38 \mid 39)$ with the assumption $e_{2}=0$, gives the frequency equation

$$
-R_{A}(\kappa) \cosh \left(\frac{B_{1} H}{\Theta}\right)+\Gamma \sqrt{2} \Omega^{-1} \delta\left(A_{1}+A_{2}\right) B_{1} \sinh \left(\frac{B_{1} H}{\Theta}\right)=0,
$$

which clearly possesses the structure 40. As Fig 13 shows, this equation is remarkable in that it exhibits an infinite number of branches, which is unexpected for Love waves. Looking at the eigenforms, we find $o_{2}=O(1)$, whence the corresponding displacement (30) blows to infinity as $\beta \rightarrow 0$. Also, although $e_{2}$ tends to zero, it combines with the exponentially large factor $B_{2}^{-1} \sinh \left(B_{2} \xi_{2}\right)=\frac{\beta}{\sqrt{2} \Theta} \sinh \left(\sqrt{2} \Theta \beta^{-1} \xi_{2}\right)$ to produce an exponentially exploding contribution. As a consequence of these observations, the dispersion relation (44) cannot be directly obtained assuming a classical solution for B and it is thereby defined as non-classical. We also observe that, to leading order, $q_{1 A}(0)=0$, which indeed corresponds to one of the boundary conditions used in Sharma and Kumar (2019); Sharma et al. (2020). However, rotation at the 
interface is finite and continuous across

$$
w_{A}^{\prime}(0)=w_{B}^{\prime}(0)=\left(A_{1}-\frac{\zeta_{12 A}}{\zeta_{11 A}} A_{2}\right) s_{1},
$$

353 354

and this condition is neglected.

\subsection{Couple stress layer perfectly bonded to a classical half-space}

Let's now consider the case when A is classical elastic and B is a couple stress layer. Then, $\kappa=O\left(\beta^{-1}\right)$ and $\Omega=O(v)$, as $\ell_{A} \rightarrow 0$ and $\beta \rightarrow \infty$, while $v \rightarrow 0$ such that $\beta v<\infty$. Thus,

$$
\begin{aligned}
& A_{1}=\beta^{-1} \sqrt{\kappa_{B}^{2}-\frac{1}{2} \beta^{2} v^{2} \Omega_{B}^{2}}+O\left(\beta^{-3}\right), \\
& A_{2}=1+\frac{\kappa_{B}^{2}}{2 \beta^{2}}+O\left(\beta^{-4}\right) \\
& B_{1}=\beta^{-1} \sqrt{\kappa_{B}^{2}-\frac{1}{2}\left(\sqrt{\left(1-\ell_{B}^{2} \Omega_{B}^{2}\right)^{2}+2 \Omega_{B}^{2}}-\left(1-\ell_{B}^{2} \Omega_{B}^{2}\right)\right)}+O\left(\beta^{-3}\right), \\
& B_{2}=\beta^{-1} \sqrt{\kappa_{B}^{2}+\frac{\Omega_{B}^{2}}{\sqrt{\left(1-\ell_{B}^{2} \Omega_{B}^{2}\right)^{2}+2 \Omega_{B}^{2}}-\left(1-\ell_{B}^{2} \Omega_{B}^{2}\right)}+O\left(\beta^{-3}\right) .}
\end{aligned}
$$

Solving (38d) lends

$$
\begin{aligned}
& s_{2}=\Gamma\left\{e_{1}\left[\left(\eta_{B}+1\right) \kappa_{B}^{2}+\frac{1}{2}\left(1-\Omega_{B}^{2} \ell_{B}^{2}-\sqrt{2 \Omega_{B}^{2}+\left(1-\Omega_{B}^{2} \ell_{B}^{2}\right)^{2}}\right)\right]\right. \\
& \left.+e_{2}\left[\left(\eta_{B}+1\right) \kappa_{B}^{2}+\frac{\Omega_{B}^{2}}{\Omega_{B}^{2} \ell_{B}^{2}-1+\sqrt{2 \Omega_{B}^{2}+\left(\Omega_{B}^{2} \ell_{B}^{2}-1\right)^{2}}}\right]\right\}+O\left(\beta^{-2}\right),
\end{aligned}
$$

which, substituted into the remaining set of boundary conditions, yields a system which is singular to leading order, owing to Eq. $39 \mathrm{~b}$ disappearing at leading order. Solving any regular subsystem gives

$$
o_{1}=o_{2}=O\left(\beta^{-1}\right), \quad \text { and } \quad s_{2}=O\left(\beta^{-2}\right),
$$

whereupon, in the limit as $\beta \rightarrow \infty$, the half-space A becomes classical and the layer $\mathrm{B}$ admits the even solution only, i.e. $e_{1}=e_{2}=O(1)$. Consequently, we expect neither rotation nor couple stress in the layer B to leading order at the bonding interface $\xi_{2}=0$, for they arise from the odd part of the solution. 


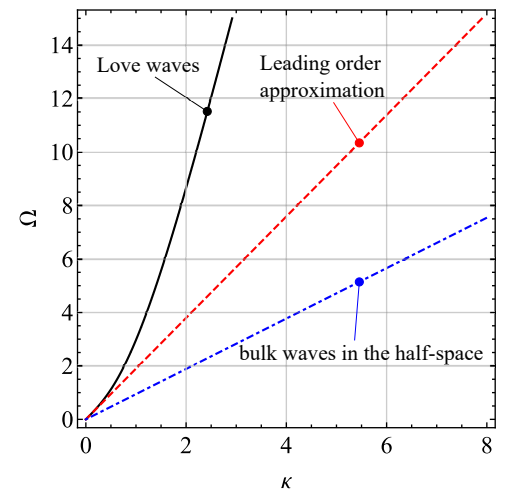

Figure 14: Love wave spectrum when $\mathrm{A}$ is classical elastic (solid, black), superposed onto the leading order approximation 49 (dashed, red) for $H_{B}=10, \eta_{B}=0.8$ and $\ell_{B}=0.2$. Bulk waves in the classical half-space $\Omega_{B}-\sqrt{2}(\beta v)^{-1} \kappa_{B}=0$ are also shown (blue, dot-dashed), with $\beta v=1.5$

Equally, no rotation arises from the half-space A either, because, at $\xi_{2}=0$, it is simply given by

$$
A_{1} s_{1}+A_{2} s_{2}=O\left(\beta^{-1}\right)
$$

Thus, rotation continuity is trivially satisfied at leading order. Looking back at couple stress continuity (46, this appears compatible with the last of Eqs. 477 inasmuch as the leading term drops out. Since this is indeed the case, couple stress continuity is also trivially satisfied. Therefore, we are left with the classical system A in perfect contact with the even solution of the couple stress layer B subject to the conditions 38a 38c 39a . Letting $H_{B}=\beta^{-1} H$, we get the novel frequency equation

$$
\zeta_{11 B} B_{1} \sinh \left(\sqrt{2} H_{B} \beta B_{1}\right)-\zeta_{12 B} B_{2} \sinh \left(\sqrt{2} H_{B} \beta B_{2}\right)=0,
$$

with the understanding that Eqs. $45 \mathrm{c}$ 45d are used to eliminate $\beta$. It is observed that terms coming from the half-space A are not present in (48) because they 3 factor out and never contribute a real wavenumber. In this condition, Love waves are always supported as in Fig 14 , which also illustrates the leading order approximation

$$
\Omega_{B}^{2}=\sqrt{\alpha_{1}} \kappa_{B}^{2}, \quad \alpha_{1}=\sqrt{2} \frac{1-\left(\eta_{B}+1\right) \frac{\sinh \left(\sqrt{2} H_{B}\right)}{\sqrt{2} H_{B}}}{1-\frac{\sinh \left(\sqrt{2} H_{B}\right)}{\sqrt{2} H_{B}}} .
$$




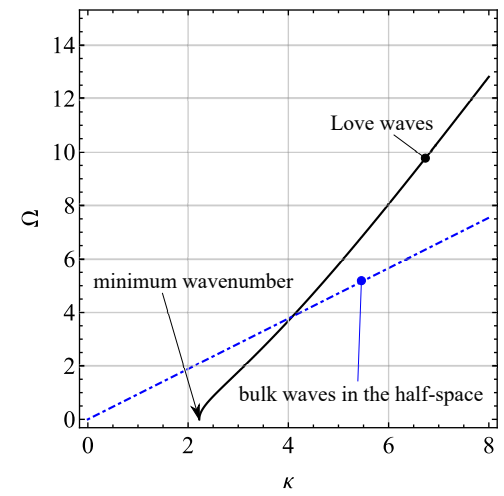

Figure 15: Love wave spectrum when $\mathrm{A}$ is classical elastic (solid, black), for $H_{B}=2, \eta_{B}=$ -0.8 and $\ell_{B}=0.2$. Bulk waves in the classical half-space $\Omega_{B}-\sqrt{2}(\beta v)^{-1} \kappa_{B}=0$ are also shown (blue, dot-dashed), with $\beta v=1.5$

With the help of this approximation, it may be shown that, if we can find real solutions of

$$
\frac{\sinh \left(\sqrt{2} H_{B}\right)}{\sqrt{2} H_{B}}=\frac{1}{1+\eta_{B}},
$$

then we get a minimum wavenumber for Love wave propagation, as in Fig 15 .

It is worth pointing out that various subsets of boundary conditions have been adopted in the literature. As an example, in Fan and Xu (2018), a couple stress layer is perfectly bonded to a classical half-space and the conditions (38a 38c 39) alongside $q_{1 A}=0$ are used instead. Since rotation continuity is missing, the resulting frequency equation does not match (48).

\section{Conclusions}

We show that incorporating microstructure into the material description leads to a nonclassical band structure for Love wave propagation, which may be conveniently back-processed for non destructive testing (NDT) evaluation of the material microstructure. In particular, an explicit expression is given for the propagation band limits, namely the cut-on and cut-off points. These are shown to correspond to the condition when the localized wave moves with the same speed as the SH bulk wave in the substrate. As a consequence, energy leaks to infinity and it is no longer confined (internal resonance). Precisely 
this condition may be taken advantage of to assess the mechanical properties of the two materials by non destructive testing. Indeed, it is simple matter to experimentally locate cut-on/cut-off frequencies and therefrom compute the relevant microstructural parameters. In general, the qualitative features of the band pattern are related to the degree of similarity between the mechanical properties of the layer and of the substrate.

Furthermore, to warrant uniqueness of the inverse problem, multiple observables needs to be collected, the easiest being the Rayleigh spectrum. In this context, we show that the frequency equation for Love waves possesses an elegant three term structure, where Rayleigh and Rayleigh-Lamb modes for a free and free/clamped layer play an important role. Therefore, consideration of Rayleigh waves besides Love waves is able to minimize the non-uniqueness connected to signal back-processing on the surface.

Finally, we consider the special situation, well represented in the literature, as either the substrate or the layer turns classical and therefore has no microstructure. We show that such glueing of dissimilar material models generally leads to inconsistencies. Indeed, when the layer microstructure vanishes, the resulting displacement field grows unbounded. In contrast, the case when the substrate microstructure becomes exceedingly small still leads to a meaningful solution, which may be obtained directly proceeding from the right set of boundary conditions. A novel dispersion relation is obtained which lends a single continuous branch with neither cut-on nor cut-off, as for classical Love waves.

\section{Acknowledgements}

The authors acknowledge financial support from POR FESR 2014-2020 ASSE 1 AZIONE 1.2.2, Project IMPReSA, CUP E81F18000310009. 


\section{Appendix A. Appendix}

\section{Appendix A.1. Coupling coefficient}

The coupling term in the dispersion relation 40 may be rewritten as

$$
\begin{aligned}
& D_{1}(\kappa)=c_{c}\left[\cosh \left(\frac{H B_{1}}{\Theta}\right) \cosh \left(\frac{H B_{2}}{\Theta}\right)-1\right] \\
& +s_{s} B_{1}^{-1} B_{2}^{-1} \sinh \left(\frac{H B_{1}}{\Theta}\right) \sinh \left(\frac{H B_{2}}{\Theta}\right)+\left(A_{1}+A_{2}\right)\left(\zeta_{11 B}-\zeta_{12 B}\right) \times \\
& {\left[s_{c} B_{1}^{-1} \sinh \left(\frac{H B_{1}}{\Theta}\right) \cosh \left(\frac{H B_{2}}{\Theta}\right)-c_{s} B_{2}^{-1} \cosh \left(\frac{H B_{1}}{\Theta}\right) \sinh \left(\frac{H B_{2}}{\Theta}\right)\right],}
\end{aligned}
$$

with the coefficients

$$
\begin{aligned}
& c_{c}=2 \zeta_{11 B} \zeta_{12 B}\left(\zeta_{11 B}+\zeta_{12 B}\right)\left(A_{1} A_{2}-\kappa^{2} \eta_{A}\right), \\
& s_{s}=-2\left(\zeta_{11 B}^{3} B_{1}^{2}+\zeta_{12 B}^{3} B_{2}^{2}\right)\left(A_{1} A_{2}-\kappa^{2} \eta_{A}\right), \\
& s_{c}=A_{1} A_{2} \zeta_{12 B}^{2}-\zeta_{11 B}^{2} B_{1}^{2}, \\
& c_{s}=A_{1} A_{2} \zeta_{11 B}^{2}-\zeta_{12 B}^{2} B_{2}^{2} .
\end{aligned}
$$

\section{Appendix A.2. Existence and uniqueness}

We consider the complex-valued function

$$
D(s)=D_{0}(s) \exp \left(-\frac{2 H}{\Theta}|s|\right),
$$

where the exponential factor is added to obtain algebraic growth as $|s| \rightarrow+\infty$. Indeed, we find that $D(s) \sim|s|^{4}$ as $|s| \rightarrow \infty$. We recall that $D(s)=D(-s)$ is central-symmetric. The path in the complex plane which is adopted to implement the argument principle is a very large circle of radius $R \rightarrow \infty$ with two pairs of loops: one circles around the branch cuts at $\pm \delta$ and the other around the branch cuts at $\pm \imath$, both being parallel to the imaginary axis, see Fig A.16. We thus see that the image of the path $\gamma$ through the function $D(s)$ circles the origin four times counter-clockwise and, possibly, two time clockwise if condition (42) is violated. In fact, this condition merely states that the image $D\left(\gamma_{\delta}\right)$ intersects the real axis to the right of the origin and contributes nothing. In this case we have four roots, a real pair and a complex pair. In contrast, if condition 


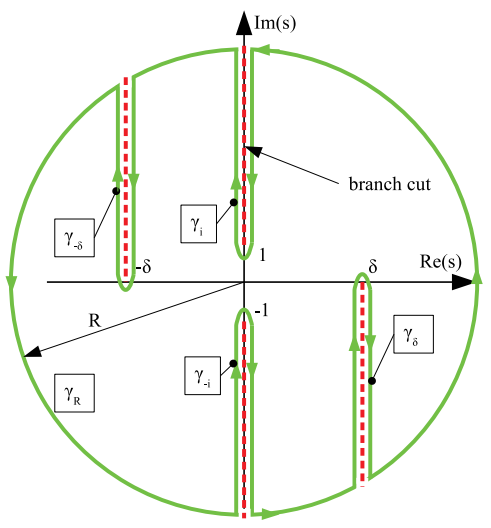

Figure A.16: Path $\gamma=\gamma_{R} \cup \gamma_{ \pm \delta} \cup \gamma_{ \pm i}$ for implementing the argument principle: the number of zeros of $D(s)$ equals the number of times the image $D(\gamma)$ winds around the origin

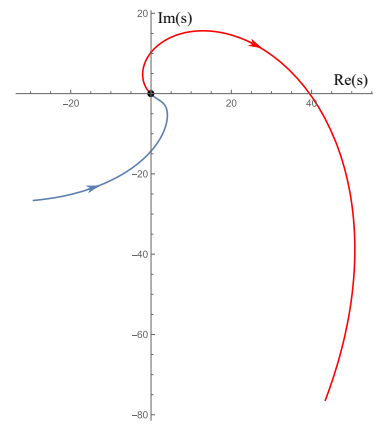

Figure A.17: The image $D\left(\gamma_{\delta}\right)$ winds around the origin in clockwise manner, because $D(\delta)<0$. Hence, the number of zeros of $D(s)$ is diminished by two 
(42) is violated, then the origin sits to the right of path, as in Fig A.17, and subtracts a pair of roots. Then, only the complex pair remains. It is finally observed that the image $D\left(\gamma_{i}\right)$ never circles the origin.

\section{References}

Achenbach, J., 1984. Wave propagation in elastic solids. volume 16 of Applied Mathematics and Mechanics. North-Holland, Elsevier.

Cagniard, L., 1962. Reflection and refraction of progressive seismic waves. McGraw-Hill.

Dal Moro, G., 2020. The magnifying effect of a thin shallow stiff layer on Love waves as revealed by multi-component analysis of surface waves. Scientific Reports 10, 1-13.

Destuynder, P., Fabre, C., 2016. Few remarks on the use of Love waves in non destructive testing. Discrete \& Continuous Dynamical Systems - S 9, 427-444.

Fan, H., Xu, L., 2018. Love wave in a classical linear elastic half-space covered by a surface layer described by the couple stress theory. Acta Mechanica 229, $5121-5132$.

Georgiadis, H., Velgaki, E., 2003. High-frequency Rayleigh waves in materials with micro-structure and couple-stress effects. International Journal of Solids and Structures 40, 2501-2520.

Gourgiotis, P., Georgiadis, H., 2015. Torsional and SH surface waves in an isotropic and homogenous elastic half-space characterized by the ToupinMindlin gradient theory. International Journal of Solids and Structures 62, $217-228$.

Gourgiotis, P., Georgiadis, H., Neocleous, I., 2013. On the reflection of waves in half-spaces of microstructured materials governed by dipolar gradient elasticity. Wave Motion 50, 437-455. 
Graff, K.F., 1991. Wave motion in elastic solids. Dover Publications Inc., New York.

Graff, K.F., Pao, Y.H., 1967. The effects of couple-stresses on the propagation and reflection of plane waves in an elastic half-space. Journal of Sound and Vibration 6, 217-229.

Jin, F., Kishimoto, K., Inoue, H., Tateno, T., 2005. Experimental investigation on the interface properties evaluation in piezoelectric layered structures by Love waves propagation, in: Key Engineering Materials, Trans Tech Publ. pp. $807-812$.

Koiter, W., 1964. Couple-stress in the theory of elasticity, in: Proc. K. Ned. Akad. Wet, North Holland Pub. pp. 17-44.

Lakes, R., 1986. Experimental microelasticity of two porous solids. International Journal of Solids and Structures 22, 55-63.

Li, Y., Wang, W., Wei, P., Wang, C., 2018. Reflection and transmission of elastic waves at an interface with consideration of couple stress and thermal wave effects. Meccanica 53, 2921-2938.

Love, A., 1911. Some problems of geodynamics. Cambridge University Press.

Maugin, G., 1988. Shear horizontal surface acoustic waves on solids, in: Recent developments in surface acoustic waves. Springer, pp. 158-172.

Mindlin, R.D., 1964. Micro-structure in linear elasticity. Archive for Rational Mechanics and Analysis 16, 51-78.

Mishuris, G., Piccolroaz, A., Radi, E., 2012. Steady-state propagation of a mode III crack in couple stress elastic materials. International Journal of Engineering Science 61, 112-128.

Nobili, A., Radi, E., Signorini, C., 2020. A new rayleigh-like wave in guided propagation of antiplane waves in couple stress materials. Proceedings of the Royal Society A 476, 20190822. 
Nobili, A., Radi, E., Vellender, A., 2019. Diffraction of antiplane shear waves and stress concentration in a cracked couple stress elastic material with micro inertia. Journal of the Mechanics and Physics of Solids 124, 663-680.

Nobili, A., Volpini, V., Signorini, C., 2021. Antiplane stoneley waves propagating at the interface between two couple stress elastic materials. Acta Mechanica , 1-19.

Noble, B., 1958. Methods based on the Wiener-Hopf technique for the solution of partial differential equations, International Series of Monographs on Pure and Applied Mathematics. Vol. 7. Pergamon Press, New York.

Ottosen, N.S., Ristinmaa, M., Ljung, C., 2000. Rayleigh waves obtained by the indeterminate couple-stress theory. European Journal of Mechanics-A/Solids 19, 929-947.

Ray, A., Singh, A., 2020. Love-type waves in couple-stress stratum imperfectly bonded to an irregular viscous substrate. Acta Mechanica 231, 101-123.

Sengupta, P., Ghosh, B., 1974. Effect of couple-stresses on the propagation of waves in an elastic layer. pure and applied geophysics 112, 331-338.

Sharma, V., Goyal, R., Kumar, S., 2020. Love waves in a layer with void pores over a microstructural couple stress substrate with corrugated boundary surfaces. Journal of the Brazilian Society of Mechanical Sciences and Engineering $42,1-16$.

Sharma, V., Kumar, S., 2019. Modelling of love-type waves in an elastic layer sandwiched between viscous liquid half space and size dependent couple stress substrate. Journal of Theoretical and Applied Mechanics 57.

Shodja, H., Goodarzi, A., Delfani, M., Haftbaradaran, H., 2015. Scattering of an anti-plane shear wave by an embedded cylindrical micro-/nano-fiber within couple stress theory with micro inertia. International Journal of Solids and Structures 58, 73-90. 
513 Vardoulakis, I., Georgiadis, H., 1997. Sh surface waves in a homogeneous 514 gradient-elastic half-space with surface energy. Journal of Elasticity 47, 147165.

516 Wang, C., Chen, X., Wei, P., Li, Y., 2017. Reflection and transmission of elastic $517 \quad$ waves through a couple-stress elastic slab sandwiched between two half-spaces. 518 Acta Mechanica Sinica 33, 1022-1039.

519 Yang, F., Chong, A., Lam, D., Tong, P., 2002. Couple stress based strain gradient theory for elasticity. International Journal of Solids and Structures $521 \quad 39,2731-2743$.

522 Zhang, L., Huang, Y., Chen, J., Hwang, K., 1998. The mode III full-field 523 solution in elastic materials with strain gradient effects. International Journal $\mathbf{5 2 4}$ of Fracture $92,325-348$. 